\title{
Throwing the dart blind-folded: comparison of computed tomography versus magnetic resonance imaging-guided brachytherapy for cervical cancer with regard to dose received by the 'actual' targets and organs at risk
}

\author{
Winnie Wing Ling Yip, FRCR, Joyce Siu Yu Wong, FRCR, Venus Wan Yan Lee, MSc, Frank Chi Sing Wong, FRCR, \\ Stewart Yuk Tung, FRCR \\ Department of Clinical Oncology, Tuen Mun Hospital, Tuen Mun, Hong Kong
}

\begin{abstract}
Purpose: Computed tomography (CT) is inferior to magnetic resonance imaging (MRI) in cervical tumor delineation, but similar in identification of organs at risk (OAR). The trend to over-estimate high-risk and low-risk clinical target volume (HRCTV, IRCTV) on CT can lead to under-estimation of dose received by $90 \%\left(\mathrm{D}_{90}\right)$ of the 'actual' CTV. This study aims to evaluate whether CT-guided planning delivers adequate dose to the 'actual' targets while spares the OAR similarly.

Material and methods: MRI-guided high-dose-rate image-guided brachytherapy (IGBT) was performed in 11 patients. The pre-brachytherapy CTs were retrospectively contoured to generate CT-guided plans. MRI-based contours

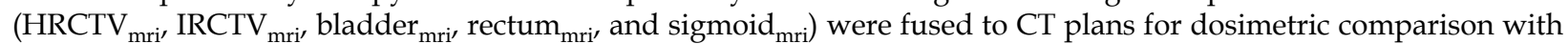
MRI-guided plans. Paired 2-tailed $t$-test and Wilcoxon signed-rank test were used to analyze data.

Results: $63.6 \%$ of CT plans achieved the HRCTV $\mathrm{mri}_{90} \mathrm{D}_{90}$ constraint ( $\geq 7.2 \mathrm{~Gy}$ in one fraction), compared with $90.9 \%$ for MRI plans. $>90 \%$ of both modalities achieved the OAR's constraints (EMBRACE). The percentage of CT and MRI plans that achieved the aims (EMBRACE II) for bladder, rectum, and sigmoid were $36.4 \%$ vs. $81.8 \%, 63.6 \%$ vs. $63.6 \%$, and $72.7 \%$ vs. $72.7 \%$, respectively. There were no statistically significant differences in $\operatorname{HRCTV}_{\text {mri }} \mathrm{D}_{90}, \operatorname{IRCTV}_{\text {mri }} \mathrm{D}_{90}$, or dose received by the most exposed $2 \mathrm{~cm}^{3}\left(\mathrm{D}_{2 \mathrm{cc}}\right)$ of $\mathrm{OAR}_{\mathrm{mri}}$ between the modalities. Excluding the CT plans not achieving HRCTV $\mathrm{mri}_{90} \mathrm{D}_{90}$ constraint, there were significant increase in bladder ${ }_{\mathrm{mri}} \mathrm{D}_{2 \mathrm{cc}}$, rectum $\mathrm{mri}_{2 \mathrm{cc}} \mathrm{D}_{2}$ and sigmoid $\mathrm{mri}_{2 \mathrm{cc}} \mathrm{D}_{2}$ compared with MRI plans (0.9 Gy/Fr, 95\% CI 0.2-1.5, $p=0.018 ; 0.9 \mathrm{~Gy} / \mathrm{Fr}, 95 \%$ CI 0.3-1.4, $p=0.009 ; 0.5 \mathrm{~Gy} / \mathrm{Fr}, 95 \%$ CI $0.2-0.9, p=0.027$, respectively).

Conclusions: MRI-based IGBT remains the gold standard. CT planning may compromise HRCTV $\mathrm{mri}_{90}$ or increase $\mathrm{OAR}_{\mathrm{mri}} \mathrm{D}_{2 \mathrm{cc}}$ which could decrease local control or increase treatment toxicity.

J Contemp Brachytherapy 2017; 9, 5: 446-452 DOl: https://doi.org/10.5114/jcb.2017.71050
\end{abstract}

Key words: brachytherapy, cervical cancer, CT, MRI.

\section{Purpose}

The radical treatment for locally advanced cervical cancer consists of radiotherapy with concurrent chemotherapy [1]. Pelvic brachytherapy following external beam radiotherapy is an effective way to maximize radiation dose to the tumor, while limiting dose to adjacent normal organs. 3D (three dimensional) brachytherapy planning has been introduced in the Western population for more than a decade to allow precision in localization of treatment targets and organs at risk. RetroEMBRACE, a retrospective multi-institutional observational study, has shown that magnetic resonance imaging (MRI)-based or computed tomography (CT)-based brachytherapy shows favorable local, pelvic control, and overall survival compared with 2D technique, with excellent toxicity profile [2].

MRI shows superior soft tissue delineation compared with CT, and has become the gold standard for image-guided brachytherapy (IGBT) in European coun-
Address for correspondence: Winnie Wing Ling Yip, FRCR, Department of Clinical Oncology, Tuen Mun Hospital, Tuen Mun, Hong Kong, phone: +852 2468 5038, fax: +852 2468 5047, e-mail: yip.wlwinnie@gmail.com
Received: 29.07.2017 Accepted: 19.09 .2017 Published: 30.10.2017 
tries [3]. Tumor dose-effect relationship has been well demonstrated in MRI-based brachytherapy [4]. However, routine use of MRI-guided brachytherapy remains a challenge in terms of time and financial costs, especially in regions with high incidence of the disease such as Asia [5].

Past studies have shown that CT-delineated HRCTV $\left(\mathrm{HRCTV}_{\mathrm{ct}}\right)$ is statistically different from MRI-delineated HRCTV (HRCTV $\mathrm{mri}_{\text {ri }}$, even with pre-planning MRI $[3,6,7,8,9,10]$. Such volumetric difference has translated into change in HRCTV $\mathrm{D}_{90}$, while dose to OAR show no statistical difference. Viswanathan et al. proved that $\mathrm{HRCTV}_{\mathrm{ct}} \mathrm{D}_{90}$ in CT-based plans is statistically lower than $\mathrm{HRCTV}_{\mathrm{mri}} \mathrm{D}_{90}$ in MRI guided plans, with mean dose per fraction of 6.7 Gy vs. 8.7 Gy, respectively [3]. Bhavana et al. showed similar findings, with the mean difference in dose per fraction less than $1 \mathrm{~Gy}$ [8].

However, regardless of the planning modality, it is the dose to the 'actual' targets as delineated based on MRI that is more of clinical relevance [4] yet infrequently reported. Despite the fact that the $\mathrm{D}_{90}$ of $\mathrm{HRCTV}_{\mathrm{ct}}$ tends to be lower in CT-based plans compared with HRCTV $\mathrm{mri}_{\mathrm{m}}$ $\mathrm{D}_{90}$ of MRI-based plans, the 'actual' HRCTV of concern i.e., HRCTV $\mathrm{Hri}_{\text {mi }}$ may fall into the high dose zone of CT plans. As a result, CT planning may result in similar dose delivered to the 'actual target'. On the other hand, further boost to the 'actual' HRCTV by MRI-based planning may be limited by the position of the adjacent organs at risk, which are just as clearly shown on CT compared with MRI [3]. The aim of this study is to compare the dose received by the 'actual' targets and organs at risk between CT- and MRI-based planning, in order to determine whether CT is an economical alternative to MRI in IGBT.

\section{Material and methods}

\section{Treatment overview}

Patients with biopsy proven cervical carcinoma with MRI-based IGBT performed between $1^{\text {st }}$ January 2016 and $28^{\text {th }}$ February 2017 were reviewed. All patients had undergone clinical gynecological examination and diagnostic MRI pelvis. They received radiotherapy with concurrent weekly cisplatin. External whole pelvic irradiation (WPI) using intensity-modulated radiation therapy (IMRT) technique (45 Gy in 25 fractions, 5 fractions per week) was planned with reference to the RTOG consensus guidelines [11,12]. For patients with para-aortic lymph node metastases, extended field IMRT with simultaneous intermittent boost (SIB) of 2.2 to 2.3 Gy per fraction was given to the involved lymph nodes [13]. After external radiotherapy, 4 fractions of high-dose-rate ${ }^{192}$ Ir brachytherapy were performed. Additional parametrial boost (API) was planned after the first fraction of brachytherapy.

\section{D brachytherapy for cervical cancer with MRI-planning}

MRI guided IGBT with 'hybrid' technique was adopted [14]. At week 5, pre-planning MRI pelvis was performed without applicator in-situ to assist treatment planning. Four fractions of brachytherapy were given in 2 weeks, with 2 fractions given on 2 consecutive days per week, leaving the applicator in-situ overnight after the first fraction of each week. Planning was done under the guidance of a pre-brachytherapy MRI with applicator in-situ during the first fraction, while CT-based planning was performed in each of the 3 remaining fractions with fused MRI contours. Adjustments were made based on change in anatomical positions of OAR. Contouring was performed according to the GEC-ESTRO (Groupe Europeen de Curiethérapie European Society for Radiotherapy and Oncology) recommendations on the planning images, with reference to the gynecological examination findings at diagnosis and during brachytherapy, diagnostic and week 5 pre-planning MRI $[15,16]$. Plan was optimized by medical physicist using Oncentra BrachyTreatment Planning System version 4.5.2 (Nucletron, an Elekta company, Elekta $\mathrm{AB}$, Stockholm, Sweden). A pre-brachytherapy CT was also done immediately after MRI during the first fraction.

\section{Details of pre-planning MRI, pre-brachytherapy $M R I$, and pre-brachytherapy CT}

MRI images were performed using a whole-body MRI system (Philips Achieva 3.0TX, Philips Healthcare, Best, The Netherlands) with 6 channel cardiac coil, with section thickness of $3 \mathrm{~mm}$ without intersection gap. Para-axial, para-coronal, and para-sagittal images were performed according to the alignment of uterus for week 5 pre-planning MRI, and according to the axis of applicator for the pre-brachytherapy MRI. For pre-planning MRI, plain T1and T2-weighted, with or without gadolinium contrast T1-weighted images were done. For pre-brachytherapy MRI, only T2-weighted plain images were performed [17]. For pre-brachytherapy CT, plain images were performed with $1.5 \mathrm{~mm}$ cut. Pre-brachytherapy CT was done within 30 minutes after pre-brachytherapy MRI.

\section{Planning aims and constraints}

Planning aims and constraints are listed in Table 1. They were derived based on the assumption that roughly the same dose was to be delivered per fraction. The planning constraints were set with reference to the EMBRACE protocol and the book "Gynecologic Radiation Therapy" [18], whereas the planning aims were similar to the updated EMBRACE II protocol, with adjustments based on retroEMBRACE and EMBRACE studies results [2,4,19] (https://www.embracestudy.dk/) from January to May 2016, only the planning constraints were followed. Planning aims were added since June 2016 in MRI-based planning. HRCTV $D_{90}$ of $\geq 85$ Gy and IRCTV $D_{90}$ of $\geq 65 \mathrm{~Gy}$ in total correspond to 3-year local control rates of $>86 \%$ [4].

\section{Data analysis}

The first fraction of IGBT was evaluated. The prebrachytherapy CT at first IGBT was contoured with reference to clinical examination at diagnosis and during the first IGBT, MRI at diagnosis, and a week 5 pre-planning MRI. CT contouring guidelines was referred [7]. There was no fusion of any MRI images to planning CT. Graphically optimized CT-based plan was generated by medical physicist based on planning aims, constraints 
Table 1. Planning aims and constraints

\begin{tabular}{|c|c|c|c|c|}
\hline Parameters & $\begin{array}{c}\text { Planning aim, } \\
\text { dose per fraction (Gy) }\end{array}$ & $\begin{array}{l}\text { Planning aim, } \\
\text { total dose (Gy) }\end{array}$ & $\begin{array}{l}\text { Planning constraint, } \\
\text { dose per fraction (Gy) }\end{array}$ & $\begin{array}{c}\text { Planning constraint, } \\
\text { total dose (Gy) }\end{array}$ \\
\hline HRCTV $D_{90}$ & $\geq 7.8$ & $\geq 90$ & $\geq 7.2$ & $\geq 85$ \\
\hline IRCTV $D_{90}$ & $\geq 4.4$ & $\geq 65$ & $\geq 3.5$ & $\geq 60$ \\
\hline Bladder $D_{2 c c}$ & $\leq 5.4$ & $\leq 80^{*}$ & $\leq 6.2$ & $\leq 90^{\#}$ \\
\hline Rectum $D_{2 c c}$ & $\leq 3.9$ & $\leq 65^{*}$ & $\leq 4.9$ & $\leq 75^{\#}$ \\
\hline Sigmoid $D_{2 c c}$ & $\leq 4.4$ & $\leq 70^{*}$ & $\leq 4.9$ & $\leq 75^{\#}$ \\
\hline
\end{tabular}

$H R C T V$, IRCTV - high-risk and low-risk clinical target volume, $D_{90}$ - the percentage of the prescribed dose received by $90 \%$ volume of the prostate, $D_{2 c c}-$ minimum dose to the most exposed $2 \mathrm{~cm}^{3}$

*With reference to EMBRACE II protocol

\#With reference to EMBRACE protocol

of tumor, and OAR as mentioned above. Oncologist, radiographer, and medical physicist were all blinded from pre-brachytherapy MRI images and MRI based contours during CT planning. Afterwards, pre-brachytherapy MRI images were fused with planning CT by rigid registration using MIM Vista version 6.0.6 based on the geometry of applicator. MRI-based contours $\left(\mathrm{HRCTV}_{\text {mri }}\right.$ IRCTV $_{\text {mri }}$

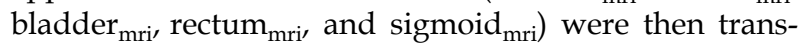
ferred to the CT images. HRCTV ${ }_{m r i} D_{90}, I_{R C T V}{ }_{m r i} D_{90}$ bladder $_{m r i} D_{2 c c}$ rectum mri $_{2 c c}$ and sigmoid $D_{m r i} D_{2 c c}$ of the CT-guided plans were compared with that of the MRI-guided plans.

\section{Statistical methods}

Data were analyzed using the SPSS software version 18. Paired 2 tailed $t$-test was used to analyze data that were in normal distribution. Wilcoxon signed-rank test was used to analyze data otherwise. The differences in HRCTV $_{\text {mri }} \mathrm{D}_{90}, \mathrm{IRCTV}_{\text {mri }} \mathrm{D}_{90}$, bladder $_{\text {mri }} \mathrm{D}_{2 \mathrm{cc}}$ rectum${ }_{\text {mri }} D_{2 c c^{\prime}}$ and sigmoid ${ }_{m r i} D_{2 c c}$ of CT-guided plans versus MRI-guided plans were compared. The patients whose HRCTV $_{\text {mri }} \mathrm{D}_{90}$ fulfilled planning constraint in both CT and MRI guided plans were selected, and the differences in $\mathrm{OAR}_{\mathrm{mri}} \mathrm{D}_{2 \mathrm{cc}}$ between the 2 plans were analyzed again. $P$ values $<0.05$ were considered significant.

\section{Results}

Between $1^{\text {st }}$ January 2016 and $28^{\text {th }}$ February 2017, 11 patients had MRI guided brachytherapy as mentioned above. The median patient age was 59 , with a range of 28 to 66 . Of the 11 patients, 1 (9.1\%) had Fédération Internationale de Gynécologie et d'Obstetrique (FIGO) stage IIA, $5(45.5 \%)$ had stage IIB, $2(18.2 \%)$ had stage IIIA, and $3(27.3 \%)$ had stage IIIB disease. Nine $(81.8 \%)$ patients had squamous cell carcinoma, 2 (18.2\%) had adenocarcinoma. The median treatment time was 49 days. Nine $(81.8 \%)$ patients had WPI. Two (18.2\%) patients, with contrast CT confirmed para-aortic lymph node metastases, received extended field radiotherapy with SIB (simultaneousintegrated boost). Ten (90.9\%) patients received API 10 Gy in 5 fractions after WPI. All patients had concurrent cisplatin, with a median of 5 doses. Utrecht applicator $(\mathrm{Nu}-$ cletron, an Elekta company, Elekta AB, Stockholm, Sweden) was used in $10(90.9 \%)$ patients, while multichannel was applied in the remaining patient. Interstitial needles were inserted in $8(72.7 \%)$ patients, among which the median number of needles was 2 (range, 1 to 7 ).

Table 2 shows the comparison of HR-CTV dimensions and volumes between HRCTV ct $_{\text {and }}$ HRCTV $\mathrm{Hri}_{\text {. There }}$ was statistically significant overestimation of width, thickness, and volume in HRCTV ${ }_{\mathrm{ct}}$. The difference between height of HRCTV $\mathrm{ct}_{\mathrm{c}}$ and HRCTV $\mathrm{Hri}_{\text {mas }}$ wot significant.

The dosimetric differences of $\mathrm{HRCTV}_{\mathrm{mri}} \mathrm{D}_{90}$ and $\mathrm{OAR}_{\text {mri }} \mathrm{D}_{2 \mathrm{cc}}$ between CT and MRI-guided plans were statistically insignificant (Table 3). Four (36.4\%) of the CT plans failed to achieve the HRCTV $\mathrm{Hri}_{\text {mi }} \mathrm{D}_{90}$ planning constraint, compared with $1(9.1 \%)$ of that of MRI plans. Five $(45.5 \%)$ of the CT plans had HRCTV $\mathrm{mri} \mathrm{D}_{90}$ higher than

Table 2. Comparison of high-risk clinical target volume (HR-CTV) dimensions and volumes between computed tomography-based and magnetic resonance imaging-based contouring

\begin{tabular}{lcccc} 
Parameters & HRCTV $_{\mathrm{ct}}{ }^{*}$ & HRCTV $_{\text {mri }}{ }^{*}$ & $\begin{array}{c}\text { Mean difference between HRCTV } \\
\text { and HRCTV }\end{array}$ mri $^{*}$ & $p$ \\
\hline Width $(\mathrm{cm})$ & $5.2(0.9)$ & $4.4(1.0)$ & $0.7(0.3$ to 1.2$)$ & 0.004 \\
\hline Thickness $(\mathrm{cm})$ & $3.8(0.6)$ & $3.1(0.7)$ & $0.7(0.3$ to 1.1$)$ & 0.001 \\
\hline Height $(\mathrm{cm})$ & $3.4(1.0)$ & $3.5(1.0)$ & $-0.2(-0.6$ to 0.2$)$ & 0.372 (NS) \\
\hline Volume $\left(\mathrm{cm}^{3}\right)$ & $50.7(23.8)$ & $33.2(20.6)$ & $17.5(9.7$ to 25.4$)$ & 0.001
\end{tabular}

HRCTV - high-risk clinical target volume

*Presented as mean (standard deviation)

\#Presented as mean (95\% confidence interval) 
Table 3. Dosimetric differences of magnetic resonance imaging (MRI)-based targets and organs at risk between computed tomography (CT)-based and MRI-based planning

\begin{tabular}{lcccc} 
Parameters & CT-guided plans* & MRI-guided plans* & $\begin{array}{c}\text { Difference between CT- } \\
\text { and MRI-guided plans }\end{array}$ & $p$ \\
\hline HRCTV $_{\text {mri }} \mathrm{D}_{90}$ & $8.1(1.9)[5.7-12.1]$ & $7.8(0.4)[7-8.3]$ & $0.3(-0.9$ to 1.4$)$ & 0.625 (NS) \\
\hline Bladder $_{\text {mri }} \mathrm{D}_{2 c c}$ & $5.5(0.9)[3.3-6.8]$ & $5.2(0.8)[4.0-7.0]$ & $0.3(-0.3$ to 1.0$)$ & 0.285 (NS) \\
\hline Rectum $_{\text {mri }} \mathrm{D}_{2 c c}$ & $3.6(1.1)[1.6-5.6]$ & $3.1(1.1)[2.1-4.9]$ & $0.4(-0.8$ to 1.0$)$ & 0.090 (NS) \\
\hline Sigmoid $_{\text {mri }} \mathrm{D}_{2 c c}$ & $3.5(1.2)[1.5-4.9]$ & $3.3(1.4)[1.1-4.7]$ & $0.2(-0.3$ to 0.7$)$ & 0.385 (NS)
\end{tabular}

HRCTV - high-risk clinical target volume, $D_{90}$ - the percentage of the prescribed dose received by $90 \%$ volume of the prostate, $D_{2 c c}-$ minimum dose to the most exposed $2 \mathrm{~cm}^{3}$

*Dose per fraction of IGBT (Gy), presented as mean (standard deviation) [range] \#Dose per fraction of IGBT (Gy), presented as mean (95\% confidence interval)

8.3 Gy per fraction (extrapolated total dose to HRCTV $\mathrm{mri}_{\mathrm{mi}}$ $>95 \mathrm{~Gy}$ ), while none of the MRI guided plans showed such overdose. With regard to EMBRACE, 1 (9.1\%) of the plans in both modalities had bladder ${ }_{\text {mri }} \mathrm{D}_{2 \mathrm{cc}}$ higher than constraint (7 Gy in the MRI plan, 6.8 Gy in the CT plan, from 2 different patients). One (9.1\%) of the CT plans showed rectum $\mathrm{mri}_{2 \mathrm{cc}}$ of $5.6 \mathrm{~Gy}$, which exceeded the constraint, compared with none in the MRI plans. No plans in either modality exceeded the constraint to sigmoid $\mathrm{mri}_{\mathrm{m}}$ $\mathrm{D}_{2 \mathrm{cc}}$. With regard to the stricter EMBRACE II protocol, $7(63.6 \%)$ of the CT plans breached the aim for bladder ${ }_{\text {mri }} \mathrm{D}_{2 \mathrm{cc}}$ compared with $2(18.2 \%)$ of the MRI plans. Four $(36.4 \%)$ of both CT and MRI plans showed overdose of rectum $\mathrm{mri}_{\text {2cc }} \mathrm{D}$. Three (27.3\%) of both CT and MRI plans exceeded the aim for sigmoid ${ }_{\text {mri }} D_{2 c c}$ (Figure 1).

The dosimetric differences in OAR were analyzed again after exclusion of the four patients whose CT-guided plans failed to achieve the HRCTV $D_{90}$ constraints (Table 4). There was significant increase in $\mathrm{OAR}_{\mathrm{mri}} \mathrm{D}_{2 \mathrm{cc}}$ in CT plans, with the overall $95 \% \mathrm{CI}$ range of 0.2 to $1.5 \mathrm{~Gy}$ per fraction. Extrapolating the results of one fraction to the whole course of IGBT, converted to EQD 2 $(\alpha / \beta=3 \mathrm{~Gy})$ the $95 \%$ confidence interval of increase in bladder $\mathrm{D}_{2 \mathrm{cc}}$ rectum $\mathrm{D}_{2 \mathrm{cc}}$ and sigmoid $\mathrm{D}_{2 \mathrm{cc}}$ by planning with CT would be from 0.5 to $5.4 \mathrm{~Gy}, 0.8$ to $4.9 \mathrm{~Gy}$, and 0.5 to $2.8 \mathrm{~Gy}$, respectively.

\section{Discussion}

This study is one of the few that have compared dose to actual targets (as represented by MRI con- tours) in CT versus MRI-guided brachytherapy. As opposed to past data showing statistically lower dose to HRCTV $_{\text {ct }} \mathrm{D}_{90}$ by CT planning compared with HRCTV $\mathrm{Hri}_{\text {mi }}$ $\mathrm{D}_{90}$ by MRI planning, the current study shows no statistical difference in HRCTV $\mathrm{mri}_{\text {mi }} \mathrm{D}_{90}$ between CT and MRI-guided plans. St-Amant et al. shows the same results in 5 patients with stage IB-IIB cervical cancer treated with intracavity brachytherapy without interstitial needle insertion [20].

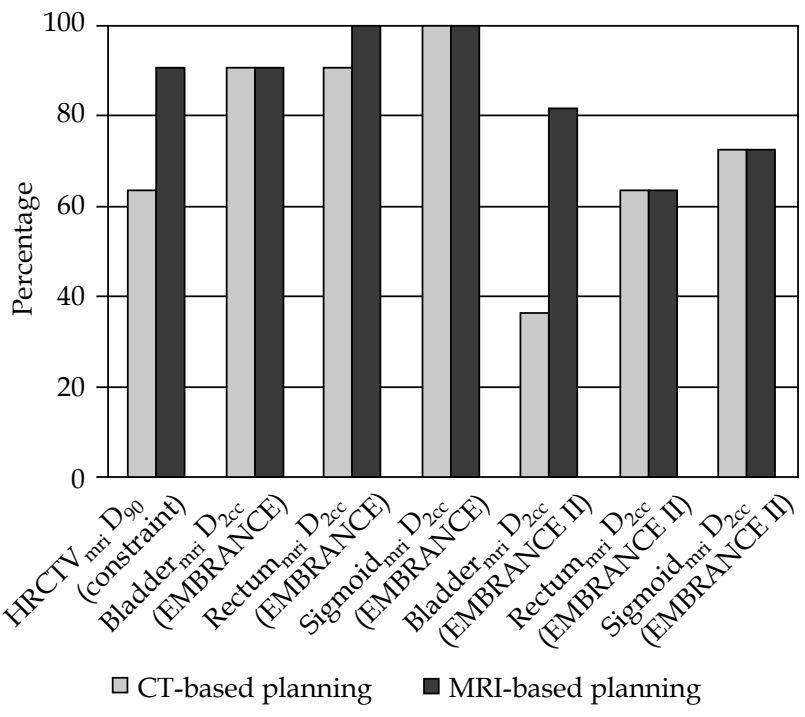

Fig. 1. Percentage of patients achieving planning constraints and aims in computed tomography- versus magnetic resonance imaging-based planning

Table 4. Dosimetric differences of organs at risk between computed tomography (CT)-based versus magnetic resonance imaging (MRI)-based planning, after exclusion of patients whose CT-based plans did not achieve HRCTV $_{\text {mri }} D_{90}$ constraint

\begin{tabular}{lcccc} 
Parameters & CT-guided plans* & MRI-guided plans* & $\begin{array}{c}\text { Difference between CT- } \\
\text { and MRI-guided plans }\end{array}$ & $p$ \\
\hline Bladder $_{\text {mri }} \mathrm{D}_{2 c c}$ & $5.9(0.6)$ & $5(0.3)$ & $0.9(0.2$ to 1.5$)$ & 0.018 \\
\hline Rectum $_{\text {mri }} \mathrm{D}_{2 c c}$ & $3.6(1.1)$ & $2.8(1.0)$ & $0.9(0.3$ to 1.4$)$ & 0.009 \\
\hline Sigmoid $_{\text {mri }} \mathrm{D}_{2 c c}$ & $4.0(1.0)$ & $3.4(1.1)$ & $0.5(0.2$ to 0.9$)$ & 0.027
\end{tabular}

HRCTV - high-risk clinical target volume, CT - computed tomography, MRI - magnetic resonance imaging, $D_{90}$ - the percentage of the prescribed dose received by $90 \%$ volume of the prostate, $D_{2 c c}-$ minimum dose to the most exposed $2 \mathrm{~cm}^{3}$

${ }^{*}$ Dose per fraction of IGBT (Gy), presented as mean (standard deviation)

\#Dose per fraction of IGBT (Gy), presented as mean (95\% confidence interval) 
However, the range of $\mathrm{HRCTV}_{\mathrm{mri}} \mathrm{D}_{90}$ in CT guided plans is excessively large (5.7 to 12.1 ). This can be explained by two possible case scenarios (Figures 2A-B). In Figure 2A,

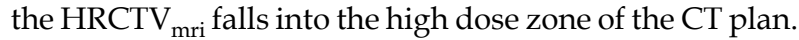
During CT-guided planning, only the $\mathrm{HRCTV}_{\mathrm{ct}}$ is known to oncologist and medical physicist. The IGBT plan is optimized to achieve the highest $\mathrm{HRCTV}_{\mathrm{ct}} \mathrm{D}_{90}$, limited by dose to OAR, which is the rectum in the example. The apparent $H R C T V_{c t} D_{90}$ is less than $7 \mathrm{~Gy}$, which fails to achieve the constraint. However, the $\mathrm{HRCTV}_{\text {mri }} \mathrm{D}_{90}$ receives a much higher dose of more than 8.3 Gy. In Figure 2B, since the $\mathrm{HRCTV}_{\mathrm{mri}} \mathrm{D}_{90}$ falls into the dose gradient zone of the CT plan, the HRCTV $\mathrm{mri} \mathrm{D}_{90}$ is under-dosed. The real life examples of the scenarios are demonstrated in Figures $3 \mathrm{~A}$ and B. In the present study, 4 out of 11 of CT-guided plans failed to achieve $\mathrm{HRCTV}_{\text {mri }}$ constraint.

A

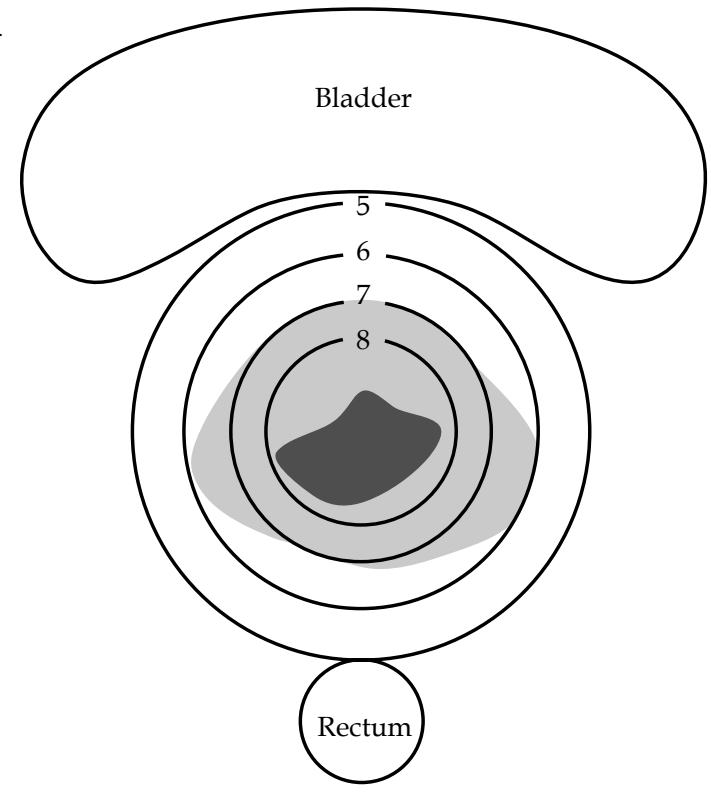

$\square$ HRCTV $_{\text {ct }} \quad \square$ HRCTV $_{\text {mri }}$
This is worrying since it correlates to inferior local control [4]. In fact, recently published data comparing MRI-guided versus CT-guided brachytherapy shows inferior overall survival for patients with CT-guided brachytherapy, which could possibly be explained by the results of the current study [21]. Five out of 11 of CT-guided plans had HRCTV $_{\text {mri }} \mathrm{D}_{90}$ higher than $8.3 \mathrm{~Gy}$, extrapolated to total biological equivalent dose of more than 95 Gy. HRCTV $\mathrm{D}_{90}$ of 95 Gy already results in excellent tumor control, especially for small tumors, and further escalation of dose is not relevant [4], but could lead to increased dose to OAR. Only $18.2 \%$ of the CT-guided plans achieve an extrapolated dose of 85 to 95 Gy to HRCTV mri $D_{90}$. The gross uncertainties in CT-guided plans raise concern despite the statistical insignificant overall results. Further studies needs to be done to validate the insignificant

B

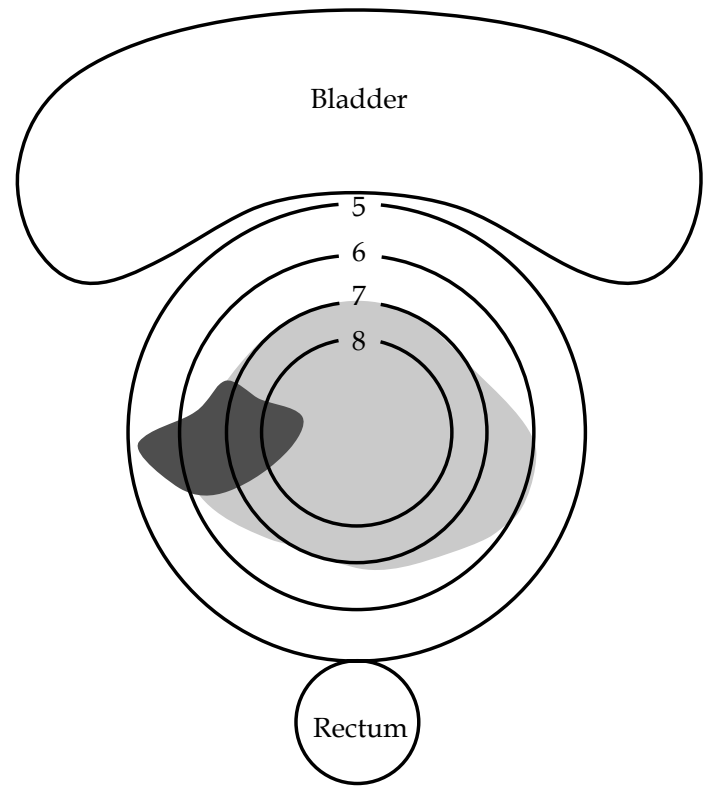

$\square$ HRCTV $_{\mathrm{ct}} \quad \square \mathrm{HRCTV}_{\mathrm{mri}}$

Fig. 2. A) $H R C T V_{\text {mri }}$ falls into the high dose zone of the computed tomography (CT)-guided plan. Although the $H R C T V_{c t} D_{90}$ is under-dosed, the actual target of interest, the $\mathrm{HRCTV}_{\text {mri }} \mathrm{D}_{90}$ receives more than $8.3 \mathrm{~Gy}$. B) $\mathrm{HRCTV}_{\text {mri }}$ falls into the dose gradient zone of the CT-guided plan. The HRCTV $\mathrm{mri}_{90} \mathrm{D}_{90}$ is under-dosed
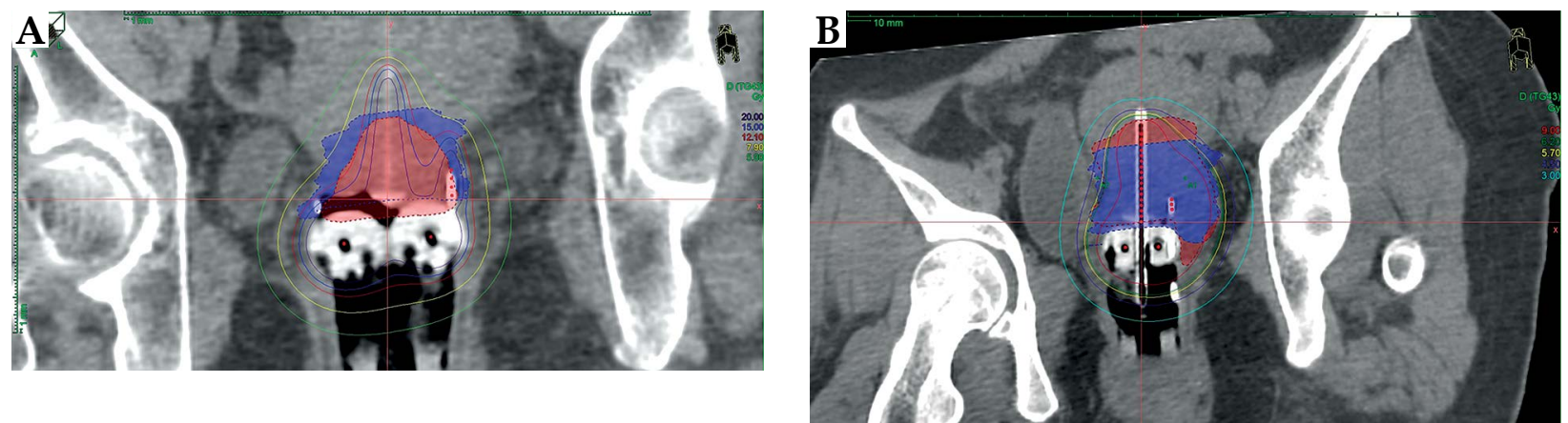

Fig. 3. A) Computed tomography (CT)-based plan of a patient with $H_{R C T V}$ shown in blue and fused $H_{R C T V}$ mri shown in red. The width of the tumor is overestimated on CT, hence HRCTV mri falls into the high dose zone. The $\mathrm{HRCTV}_{\mathrm{ct}} \mathrm{D}_{90}$ is $7.9 \mathrm{~Gy}$, but the dose delivered to the actual target, $\mathrm{HRCTV}_{\mathrm{mri}} \mathrm{D}_{90}$, is $12.1 \mathrm{~Gy}$. B) CT-based plan of another patient with $\mathrm{HRCTV}_{\mathrm{ct}}$ shown in blue color and fused $\mathrm{HRCTV}_{\mathrm{mri}}$ shown in red color. Since the height of the tumor is underestimated on CT, HRCTV $\mathrm{mri}_{\text {falls into }}$ the dose gradient zone. The HRCTV $\mathrm{Ht}_{90} \mathrm{D}_{90}$ is $6.2 \mathrm{~Gy}$, while HRCTV $\mathrm{Hri}_{\text {is }} 5.7 \mathrm{~Gy}$ 
results of current study. Studies to correlate patient, tumor, and treatment factors to the extent of dose uncertainties are also crucial to guide patient selection for MRI-guided planning in a resource limited setting.

The present study demonstrates no overall differences in $\mathrm{OAR}_{\mathrm{mri}} \mathrm{D}_{2 \mathrm{cc}}$ between the two planning modalities. Subsequently, patients that failed to achieve HRCTV ${ }_{\text {mri }}$ $\mathrm{D}_{90}$ constraint in CT plans were excluded and analysis was repeated. This analysis is designed because the cases which failed to achieve HRCTV $\mathrm{mri}_{90} \mathrm{D}_{90}$ in CT plans are clearly inferior, further studies on the OAR $\mathrm{D}_{2 \mathrm{cc}^{\prime}}$ even if more favorable than MRI-guided plans (possibly due to geographical miss of targets), is not of importance. Among patients with HRCTV ${ }_{\text {mri }} \mathrm{D}_{90} \geq 7.2 \mathrm{~Gy}$ in CT plans, there is a statistical increase in $\mathrm{OAR}_{\text {mri }} \mathrm{D}_{2 \mathrm{cc}}$. This indicates that even if CT plans fulfill the HRCTV constraints, there is a tendency to over-treat the OAR compared with MRI-guided planning.

Both EMBRACE and EMBRACE II protocols were used to analyze dose to OAR (Table 1). Since medical physicist strictly observed the EMBRACE constraints, most treatment plans in either modality achieved the requirement. However, there were more CT plans breaching the EMBRACE II requirement than MRI, with more than $60 \%$ of those breaching the bladder planning aim.

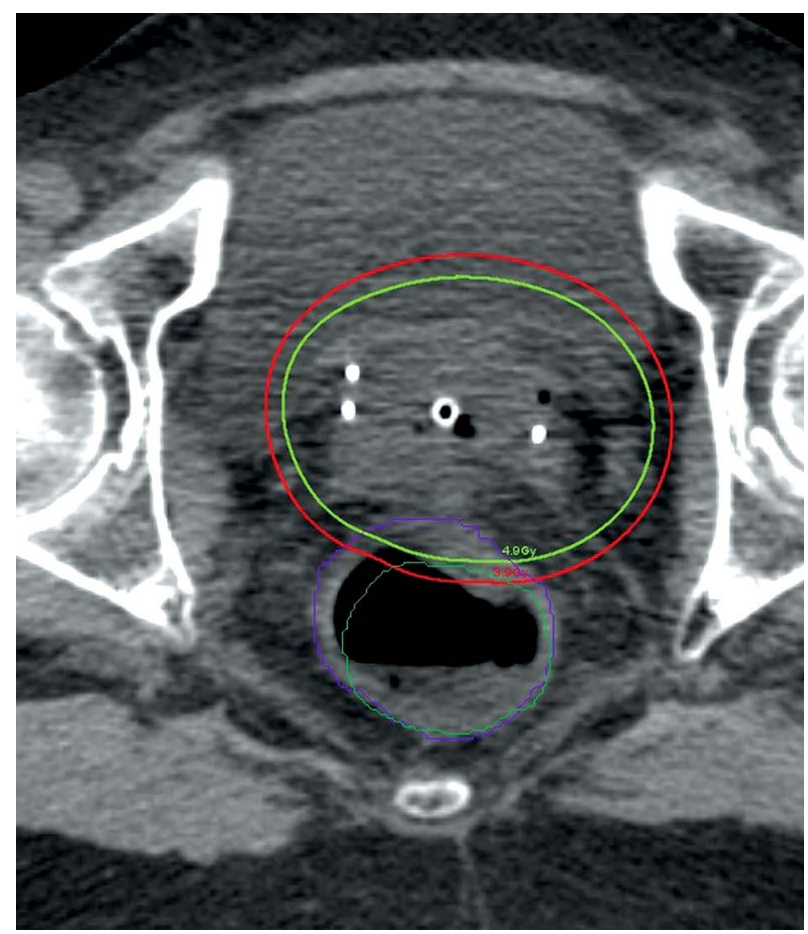

Fig. 4. The computed tomography (CT)-based rectum is contoured in purple, while the fused magnetic resonance imaging (MRI)-based rectum is contoured in green. There is a change in the anatomical position of rectum between MRI and CT images, which can be a confounding factor for the dosimetric differences in the two planning modalities. The $4.9 \mathrm{~Gy}$ isodose line is shown in light-green, while the 3.9 Gy isodose line is shown in red. They correspond to the rectum $\mathrm{D}_{2 \mathrm{cc}}$ dose constraint (EMBRACE) and aim (EMBRACE II), respectively
This bears clinical significance. Recent data have shown that the dose of rectum $\mathrm{D}_{2 \mathrm{cc}}$ correlates well with late rectal toxicities [19]. The EMBRACE II protocol also revealed the unpublished data from 680 patients, showing a significant dose-toxicity relationship at bladder $\mathrm{D}_{2 c c}$ beyond $80 \mathrm{~Gy}$, with significant increase in grade 2 or higher morbidities except ureter stenosis. The final results are awaited. The dose-toxicity relationship of sigmoid $D_{2 c c}$ is so far least established, but it is reasonable to assume characteristics similar to rectum until further data is available.

A major limitation of this study is the small sample size, given the short history of MRI-guided brachytherapy implementation in Hong Kong. Compared with 2D or CT-guided brachytherapy, MRI-based treatment involves longer time and higher financial costs, and requires intensive multi-disciplinary collaboration. Routine MRI-guided brachytherapy would remain a challenge in the years to come. Studies investigating in transrectal ultrasound or transabdominal ultrasound in combination with CT for target definition, has addressed the problem of resource limitation against utilization of MRI-planning. However, it is operator and equipment dependent, and statistically significant under-estimation of tumor thickness by transrectal ultrasound raises concern towards geographical miss $[20,22]$. To facilitate better patient selection in the era of transition from CT- to MRI-guided planning, studies with a larger scale to delineate factors that predispose to over- or under-treatment of OARs and CTVs in CT-guided planning are eagerly awaited. Patients with high body mass index and those with larger cervical tumor involving parametrium at diagnosis with good response after external radiotherapy, show the greatest discrepancy between CT- and MRI-delineated HRCTV [7,23]. This could be the direction for further studies.

There are no CT or MRI suites in the operation theatre (OT) in our center. Time is needed for patient transferal between the OT and the scanning rooms. It results in change in the anatomical positions of OARs, which are not fully correctable by image fusion based on the location of applicator (Figure 4). This could be a confounding factor in the difference between CT and MRI-guided plans.

\section{Conclusions}

MRI-guided IGBT remains the standard of care. CTbased planning could lead to an unrecognized underdose of the actual treatment target or over-dose of the OARs, which could compromise the treatment response and worsen the toxicities. Further studies to illustrate the group of patients who would benefit the most from MRI-guided planning are eagerly awaited.

\section{Acknowledgement}

Authors would like to thank Mr. Jia Jie Huang for his contribution.

\section{Disclosure}

Authors report no conflict of interest. 


\section{References}

1. Chemoradiotherapy for Cervical Cancer Meta-Analysis Collaboration. Reducing uncertainties about the effects of chemoradiotherapy for cervical cancer: a systematic review and meta-analysis of individual patient data from 18 randomized trials. J Clin Oncol 2008; 26: 5802-5812.

2. Sturdza A, Pötter R, Fokdal LU et al. Image guided brachytherapy in locally advanced cervical cancer: Improved pelvic control and survival in RetroEMBRACE, a multicenter cohort study. Radiother Oncol 2016; 120: 428-433.

3. Viswanathan AN, Dimopoulos J, Kirisits C et al. Computed tomography versus magnetic resonance imaging-based contouring in cervical cancer brachytherapy: results of a prospective trial and preliminary guidelines for standardized contours. Int J Radiat Oncol 2007; 68: 491-498.

4. Tanderup K, Fokdal LU, Sturdza A et al. Effect of tumor dose, volume and overall treatment time on local control after radiochemotherapy including MRI guided brachytherapy of locally advanced cervical cancer. Radiother Oncol 2016; 120: 441-446.

5. Harkenrider MM, Shea SM, Wood AM et al. How one institution overcame the challenges to start an MRI-based brachytherapy program for cervical cancer. J Contemp Brachytherapy 2017; 9: 177-186.

6. Hegazy N, Pötter R, Kirisits C et al. High-risk clinical target volume delineation in CT-guided cervical cancer brachytherapy: impact of information from FIGO stage with or without systematic inclusion of 3D documentation of clinical gynecological examination. Acta Oncologica 2013; 52: 1345-1352.

7. Viswanathan AN, Erickson B, Gaffney DK et al. Comparison and Consensus Guidelines for Delineation of Clinical Target Volume for CT- and MR-Based Brachytherapy in Locally Advanced Cervical Cancer. Int J Radiat Oncol Biol Phys 2014; 90: 320-328.

8. Rai B, Patel FD, Tomar P et al. A study to assess the feasibility of using CT ( \pm diagnostic MRI) instead of MRI at brachytherapy in image guided brachytherapy in cervical cancer. J Radiother Pract 2014; 13: 438-446.

9. Pötter R, Federico M, Sturdza A et al. Value of magnetic resonance imaging without or with applicator in place for target definition in cervix cancer brachytherapy. Int J Radiat Oncol Biol Phys 2016; 94: 588-597.

10. Eskander RN, Scanderbeg D, Saenz CC et al. Comparison of Computed Tomography and Magnetic Resonance Imaging in Cervical Cancer Brachytherapy Target and Normal Tissue Contouring. Int J Gynecol Cancer 2010; 20: 47-53.

11. Lim K, Small Jr. W, Portelance L et al. Consensus guidelines for delineation of clinical target volume for intensity-modulated pelvic radiotherapy for the definitive treatment of cervix cancer. Int J Radiat Oncol Biol Phys 2017; 79: 348-355.

12. Small Jr. W, Mell LK, Anderson P et al. Consensus guidelines for delineation of clinical target volume for intensity-modulated pelvic radiotherapy in postoperative treatment of endometrial and cervical cancer. Int J Radiat Oncol Biol Phys 2008; 71: 428-434.

13. Ahmed RS, Kim RY, Duan J et al. IMRT dose escalation for positive para-aortic lymph nodes in patients with locally advanced cervical cancer while reducing dose to bone marrow and other organs at risk. Int J Radiat Oncol Biol Phys 2004; 60: 505-512.

14. Nesvacil N, Pötter R, Sturdza A et al. Adaptive image guided brachytherapy for cervical cancer: A combined MRI-/CT-planning technique with MRI only at first fraction. Radiother Oncol 2013; 107: 75-81.

15. Haie-Meder C, Pötter R, van Limbergen E et al. Recommendations from Gynaecological (GYN) GEC-ESTRO Working
Group (I): concepts and terms in 3D image based 3D treatment planning in cervix cancer brachytherapy with emphasis on MRI assessment of GTV and CTV. Radiother Oncol 2005; 74: 235-245.

16. Pötter R, Haie-Meder C, van Limbergen E et al. Recommendations from gynaecological (GYN) GEC ESTRO working group (II): Concepts and terms in 3D image-based treatment planning in cervix cancer brachytherapy - 3D dose volume parameters and aspects of 3D image-based anatomy, radiation physics, radio. Radiother Oncol 2006; 78: 67-77.

17. Dimopoulos JCA, Petrow P, Tanderup K et al. Recommendations from Gynaecological (GYN) GEC-ESTRO Working Group (IV): Basic principles and parameters for MR imaging within the frame of image based adaptive cervix cancer brachytherapy. Radiother Oncol 2012; 103: 113-122.

18. Viswanathan AN, Kirisits C, Erickson BE et al. Gynecologic Radiation Therapy: Novel Approaches to Image-Guidance and Management. Berlin Heidelberg, Springer 2016.

19. Mazeron R, Fokdal LU, Kirchheiner K et al. Dose-volume effect relationships for late rectal morbidity in patients treated with chemoradiation and MRI-guided adaptive brachytherapy for locally advanced cervical cancer: Results from the prospective multicenter EMBRACE study. Radiother Oncol 2016; 120: 412-419.

20. St-Amant P, Foster W, Froment MA et al. Use of 3D transabdominal ultrasound imaging for treatment planning in cervical cancer brachytherapy: Comparison to magnetic resonance and computed tomography. Brachytherapy 2017; 16: 847-854.

21. Kamran SC, Manuel MM, Cho LP et al. Comparison of outcomes for MR-guided versus CT-guided high-dose-rate interstitial brachytherapy in women with locally advanced carcinoma of the cervix. Gynecol Oncol 2017; 145: 284-290.

22. Schmid MP, Nesvacil N, Pötter R et al. Transrectal ultrasound for image-guided adaptive brachytherapy in cervix cancer - an alternative to MRI for target definition? Radiother Oncol 2016; 120: 467-472.

23. Swanick CW, Castle KO, Vedam S et al. Comparison of Computed Tomography- and Magnetic Resonance Imaging-based Clinical Target Volume Contours at Brachytherapy for Cervical Cancer. Int J Radiat Oncol Biol Phys 2016; 96: 793-800. 